\title{
THE X-RAY EMITTING GALAXY GROUP SHKH 360
}

\author{
II. TIERSCH ${ }^{1,6}$, H. OLEAK ${ }^{1}$, D. STOLL ${ }^{1,6}$, A.D. SCHWOPE ${ }^{2}$, \\ S. NEIZVESTNY ${ }^{3}$, H. BÖHRINGER ${ }^{4}$ AND L. CORDIS ${ }^{5}$ \\ ${ }^{1}$ Universität Potsdam, An der Sternwarte 16, Germany \\ ${ }^{2}$ Astrophysikalisches Institut Potsdam, Potsdam, Germany \\ ${ }^{3}$ Special Astrophysical Observatory, Nizhny Arkhyz, Russia \\ ${ }^{4}$ MPI Extraterrestrische Physik, Garching, Germany \\ ${ }^{5}$ IIamburger Sternwarte, Hamburg, Germany \\ ${ }^{6}$ Visiting astronomer at the DSAZ, Spain
}

Shkh 360 has the characteristic signature of a strongly interacting group. Seven galaxics are embedded in a common extended halo and the isophotes indicate clear signs of alignment in $\mathrm{B}, \mathrm{V}$, and $\mathrm{R}$. The parameters of the group as the redshift, $z$, the distance, $d$, the projected diameter, $D$, (basing on $H=55 \mathrm{~km} / \mathrm{s} / \mathrm{Mpc}$ ), the virial radius, $R_{\text {vir }}$, the velocity dispersion, $\sigma_{v}$, the virial mass, $\mathcal{M}_{v i r}$, the crossing time, $\tau$, and the space density of galaxies, $n$, are given in the Table.

$\begin{array}{crrccccc}z & d & \sigma_{v} & D & R_{\text {vir }} & \mathcal{M}_{\text {vir }} & \mathcal{M} / \mathcal{L} & \tau \\ & {[\mathrm{Mpc}]} & {[\mathrm{km} / \mathrm{s}]} & {[\mathrm{kpc}]} & {[\mathrm{kpc}]} & {\left[10^{12} \mathcal{M}_{\odot}\right]} & {\left[\mathcal{M}_{\odot} / \mathcal{L}_{\odot}\right]} & {[\mathrm{Gyr}]} \\ 0.1082 & 590 & 258 & 250 & 77 & 5.6 & 10 & 0.122\end{array}$

The space density of the galaxies in Shkh 360 is $210^{3}$ galaxies $/ \mathrm{Mpc}^{3}$, much higher than in galaxy clusters. The interaction between the galaxies results in a hot $X$-ray emitting intracluster medium which was investigated from the ROSAT PSPC all sky survey. The gas distribution is roughly symmetric. The center of the X-ray emitting region is located about 15 arcsec north-east of the most luminous galaxy. The X-ray luminosity, $L_{x}$, of Shkh 360 amounts to $8.310^{43} \mathrm{erg} / \mathrm{s}$. The values of $L_{x}$ and $\sigma_{v}$, found for this and other Shakhbazian groups (Tiersch et al. 1994 in: H.T. MacGillivray et al.: Astronomy from Wide-Field Imaging, Kluwer, p. 623), confirm the finding that the correlation $L_{x} \sim \sigma_{v}{ }^{4}$, established for galaxy clusters (Quintana \& Melnick 1982, Astron.J. 87, 972), is also valid for galaxy groups, representing the lower end of the scale. 